\title{
Influence of a wearer's voice on noise dosimeter measurements
}

\author{
Steven Ryherd ${ }^{\text {a) }}$ and Mendel Kleiner \\ Chalmers Room Acoustic Group, Department of Applied Acoustics, Chalmers University of Technology, \\ 41296 Gothenburg, Sweden \\ Kerstin Persson Waye \\ Occupational and Environmental Medicine, Department of Public Health and Community, \\ Göteborg University, Box 414, 40530 Gothenburg, Sweden \\ Erica E. Ryherd \\ Woodruff School of Mechanical Engineering, Georgia Institute of Technology, Atlanta, Georgia, 30332-0405
}

(Received 13 January 2010; revised 30 August 2011; accepted 11 December 2011)

\begin{abstract}
In recent years, interest in personal noise exposure has expanded beyond a workplace safety measure to become an effective means of investigating physiological effects of the acoustic environment on an individual. This work investigates the effects of the wearer's voice as a possible dominant sound source on body-mounted noise dosimeters and develops methods to improve the application of dosimeter measurements in medium-level noise environments. Subjects experienced a controlled set of acoustic conditions while wearing a dosimeter. In each condition, sound pressure levels were recorded with and without the subject speaking controlled phrases. Three experimental variables were considered-room type, noise type, and noise level. All three variables had a statistically significant effect upon the contribution of speech to a dosimeter measurement; for example, noise level was shown to cause a change in speech contribution by as much as $5.5 \mathrm{~dB}$ between sequential levels. Based upon the analysis, a method of predicting the decibel contribution of a wearer's voice was developed. The results of this study can be used to estimate the effect of a wearer's voice on dosimeter measurements in medium-level noise environments. (C) 2012 Acoustical Society of America. [DOI: 10.1121/1.3675941]
\end{abstract}

PACS number(s): 43.50.Qp, 43.50.Yw, 43.55.Hy, 43.70.Mn [KVH]

Pages: $1183-1193$

\section{INTRODUCTION}

Noise dosimeters are sound level meters that passively monitor the sound pressure level of a designated environment. A dosimeter's negligible weight and mobility provide the convenience of continuous monitoring at the location of the individual wherever they may go. Traditionally, dosimeters are used in industrial noise environments with relatively high background noise levels where the human voice is not a dominant noise source and has very little effect on the measured levels. However, in medium-level noise environments (i.e., 40-80 dBA) like hospitals, schools and offices, the wearer's voice can become a major contributing sound source resulting in the potential for a significant increase in sound levels recorded by body-mounted noise dosimeters.

In recent years, interest in personal noise exposure has expanded beyond traditionally high-level noise environments to include medium-level noise below $80 \mathrm{dBA}^{.1-5}$ Although dosimeters are a convenient means of monitoring sound levels experienced by an individual, dosimeter levels may not be characteristic of the background noise environment due to the influence of the wearer's voice at such a close proximity. Being able to account for the effects of a wearer's voice would allow for the continued use of dosimeters to

\footnotetext{
a) Author to whom correspondence should be addressed. Electronic mail: srryherd@arpeggioacoustics.com. Current Address: Arpeggio Acoustic Consulting, LLC, 1947 Aspen Drive, NE, Atlanta, Georgia 30345.
}

characterize medium-level noise environments even when influenced by a wearer's voice. The purpose of this study is to improve the application of noise dosimeters for future use in medium-level noise environments. The two primary outcomes are to (1) determine the influence of a wearer's voice on levels measured by noise dosimeters and (2) suggest a method of accounting for speech effects when performing dosimeter measurements in practice.

\section{PREVIOUS RESEARCH}

Over the past 30 years, studies have been performed on dosimeters in laboratory and in situ environments to better understand the practical aspects of measuring personal noise exposure. Laboratory measurements were conducted to develop transfer functions to the ear for body-mounted dosimeter measurements ${ }^{6,7}$ and to investigate effects of microphone angle. ${ }^{8}$ In situ studies have also been performed to investigate the accuracy of measurements in potentially hazardous noise environments ${ }^{9-12}$ and to compare various measurement methods. ${ }^{13,14}$ However, because these studies focus on industrial noise environments with relatively high background noise levels, the speaker's voice is not considered to have a significant influence on the measurements.

Body mounted dosimeters continue to serve as an ideal instrument for recording the time-integrated noise exposure of a person in a fluctuating noise environment by eliminating the time consuming effort of a sound level meter survey. ${ }^{15}$ However, in medium-level noise environments (i.e., 40-80 dBA), 
the wearer's voice can become a major contributing sound source to the sound levels recorded by a body-mounted noise dosimeter. Depending on the purpose of the measurement, the influence of the voice may not be a desirable component of the recorded measurement.

The human voice has been shown to overcome mediumlevel background noise due to the Lombard effect. ${ }^{16-18}$ The effect, attributed to Etienne Lombard for his work published in 1911, is described as when "a speaker changes his voice level similarly when the ambient noise level increases, on the one hand, and when the level at which he hears his own voice (his sidetone) decreases, on the other...the speaker tries to maintain a speech-to-noise ratio favorable for communication."19 The result of this effect is often referred to as Lombard speech. The use of body-mounted dosimeters in medium-level noise environments when Lombard speech is present introduces the potential influence of the wearer's voice on sound field measurements. Presently, there are no means to quantify such voice contributions with respect to noise dosimeter measurements.

Previous vocal production research has addressed some aspects related to the current study. Past research includes investigations of sound levels along with analysis of spectral content, duration of speech, and intelligibility of speech while under various conditions including automobile noise, while using earplugs, and in levels ranging from $60-100 \mathrm{~dB} .^{20-22}$ Further research has focused on various means of isolating measurements of vocal effort from background noise through the use of accelerometers ${ }^{23}$ and binaural microphones. ${ }^{24}$ Another study was conducted with the intent to eliminate the influence of the wearer's voice on noise dosimeter measurements by using a two dosimeter method. ${ }^{25}$ However, the results were not published, and specifically, the measured contribution of the voice was not documented. Despite the variety of research relating to environment-dependent speech production and multiple proposed methods for isolating vocal effort, no study has published data relating the contribution of the wearer's voice to noise dosimeter measurements.

Many studies exist that investigate factors relating to dosimeter measurements or consider the effect of noise on the human voice; however, questions still remain. What is the effect of a wearer's voice on dosimeter measurements in medium-level noise environments, and how can those effects be accounted for in future dosimeter measurements?

\section{OBJECTIVES}

The purpose of this study is to improve the application of noise dosimeters for the future use in medium-level noise environments. The two primary outcomes are to (1) determine the influence of a wearer's voice on levels measured by noise dosimeters and (2) suggest a method of accounting for speech effects when performing dosimeter measurements in practice. In pursuit of the primary objectives, three characteristics are investigated as major influences on a person's perception of an acoustical environment. These three characteristics result in three secondary objectives to determine the influence of a wearer's voice on dosimeter measurements due to (1) the physical room in which measurements are made, (2) the type of background noise present, and (3) the level of the background noise. All of these characteristics became experimental variables in the design of the study and the analysis of results.

In support of the primary and secondary objectives, the measured results were used to determine an individual's speech contribution, $C_{S}$, under each condition. $C_{S}$ was defined for this study as

$$
C_{S}=L_{p A, \text { speech }}-L_{p A, \text { no speech }},
$$

where $L_{p A \text {,speech }}$ is the $A$-weighted equivalent sound pressure level, $L_{p A \text {,eq, }}$ obtained from the dosimeter during the time when the subject is speaking in a given condition and $L_{p A \text {,no speech }}$ is the $L_{p A \text {,eq }}$ value obtained from the dosimeter during the time when the subject is not speaking during a given condition.

The speech contributions from all subjects under each condition were then analyzed for statistical significance of each experimental variable. The quantity of subjects was determined to ensure statistical power while maintaining similar proportions of male and female subjects. The study was performed in Sweden. To exclude the influence of nonnative language on speech and hearing, all subjects were native Swedish speakers with all text and speaking during the measurements conducted in Swedish

\section{METHODOLOGY}

\section{A. Experimental conditions}

Three aspects of the acoustical environment were used to define each experimental condition experienced by a subject - type of room, type of noise, and noise level. Room type consisted of two rooms - "diffuse" with minimal absorption and in situ which was a typical office space with relatively more absorption than the diffuse room. Noise type consisted of two noises- "modified pink" which was slightly modified random pink noise and "canteen" which consisted of recorded conversations. Finally, noise level consisted of four mediumlevel noise levels $-45,55,65$, and $75 \mathrm{dBA} L_{\text {eq. }}$ These three experimental variables are summarized in Table I.

\section{Room type}

Measurements were recorded in two rooms-diffuse and in situ. The diffuse room served as a controlled environment with minimal absorption to reinforced reflections. The diffuse room was a $95 \mathrm{~m}^{3}$ acoustic testing chamber constructed of concrete, and it contained diffusive panels suspended from the ceiling intended to reduce modes within the space. Diffusivity in the space can be assumed down to $300 \mathrm{~Hz}$, and the associated reverberation time was $2.0 \mathrm{~s}$.

The in situ room was a closed-plan office that introduced absorption and was used to investigate the influence of a typical room upon the subject's speech. The in situ room was $81 \mathrm{~m}^{3}$ and contained multiple book shelves, lay-in absorptive ceiling tiles, linoleum flooring, a desk, a table, five lightly padded chairs, one over-stuffed leather chair, and two large windows with curtains. Reverberation time 
TABLE I. Experimental variables.

\begin{tabular}{ll}
\hline \hline Experimental variables & \\
\hline Room: & Diffuse \\
& In situ \\
Noise: & Modified pink \\
& Canteen \\
Level: & 45 \\
(dBA) & 55 \\
& 65 \\
& 75 \\
\hline \hline
\end{tabular}

measurements were conducted in the space and were found to be $0.3 \mathrm{~s}$ based on the decay curve.

\section{Noise type}

Within each room, two types of noises were presented during the measurements-modified pink and canteen. The noise type referred to as "modified pink" was computer generated using Adobe AUDITION v2.0 and acoustically controlled in its random frequency content. The signal was equalized separately for each room at the subject location to produce a modified pink spectrum - a flat frequency response for the octave bands from $125 \mathrm{~Hz}$ to $1 \mathrm{kHz}$ and approximately a $6 \mathrm{~dB}$ decrease per octave band above $1 \mathrm{kHz}$ as shown in Fig. 1.

The canteen noise resembled background noise from a restaurant including multiple conversations in Swedish, extraneous dinnerware sounds, and footsteps. The signal used for this study was compiled from multiple recordings of small groups of people having a dinner party in an anechoic room. Five independent tracks were combined using Adobe AUDITION v2.0 and the dynamic range of the signal was compressed to $20 \mathrm{~dB}$. The resulting signal ensured a more consistent level of noise throughout the canteen conditions when compared to a single conversation. The spectral content of the canteen noise is also shown in Fig. 1 and compared to the modified pink noise spectra.

\section{Noise level}

Four $A$-weighted, equivalent noise levels were selected to represent the medium-level sound pressure levels of inter-

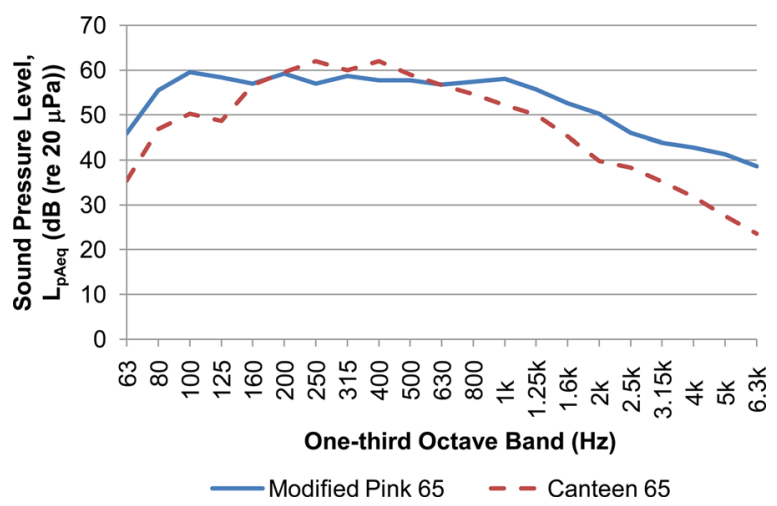

FIG. 1. (Color online) A comparison of the spectral content of the noise types presented to the subjects in each room. Shown are levels relative to the $65 \mathrm{dBA}$ noise level conditions for both modified pink and canteen noise. Each condition was adjusted accordingly for the room and noise level. est in each environment-45, 55, 65, and $75 \mathrm{dBA}$. The selected levels were below the traditionally high noise levels regulated by the United State's Occupational Safety and Health Administration (OSHA) and the Swedish Work Environment Authority. These lower levels resulted in the wearer's voice being a dominant noise source at the dosimeter microphone. The range of levels was intended to provide a wide understanding of the effect of noise level upon speech-influenced dosimeter levels.

\section{B. Test setup}

\section{Physical layout and signal presentation}

The physical layout of the diffuse and in situ room is shown in Figs. 2 and 3, respectively. The subject was positioned in both rooms at least $2.0 \mathrm{~m}$ away from any wall in an asymmetrical orientation to minimize modal influences of the room. The subject stood $1.5 \mathrm{~m}$ away from a computer monitor used to display the text for each condition. The monitor was placed at a height of approximately $1.0 \mathrm{~m}$. The position of the monitor corresponded to the location of the intended or "imagined" receiver position with whom the subject was instructed to converse.

Signals were presented in each room using a standard soundcard, amplifier, and speaker system. The soundcard used to play the sound files was an Edirol UA-101. The signal was then sent to one of the stereo integrated amplifiersNAD model 310 and 312. Each amplifier served one of the sets of four speakers in a given test room. The controlled sound in each space was produced using a set of four loudspeakers. The loudspeakers were placed on the floor and oriented asymmetrically in all four corners of the room to encourage diffusivity of the room's sound field.

Each noise type was calibrated at all four levels in both rooms using a Brüel \& Kjær 2260 Investigator, Type 1 sound level meter as specified in ANSI S1.4-2006. ${ }^{26}$ The meter was placed at the subject position- $1.5 \mathrm{~m}$ from the text screen and at a height of $1.6 \mathrm{~m}$. $L_{p A \text {,eq }}$ measurements were made in order to calibrate each condition's designed noise

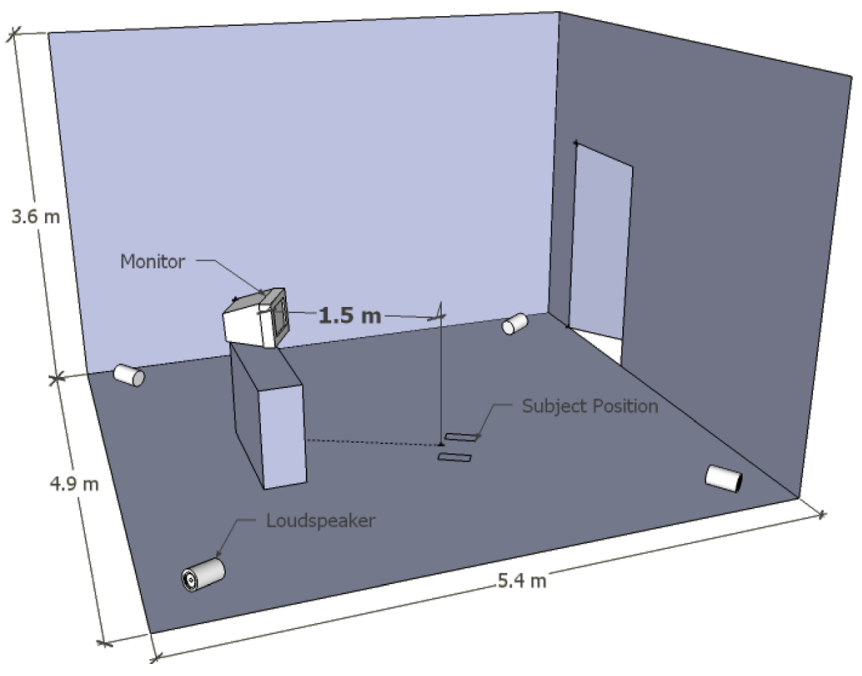

FIG. 2. (Color online) Diffuse room layout with dimensions and equipment locations. 


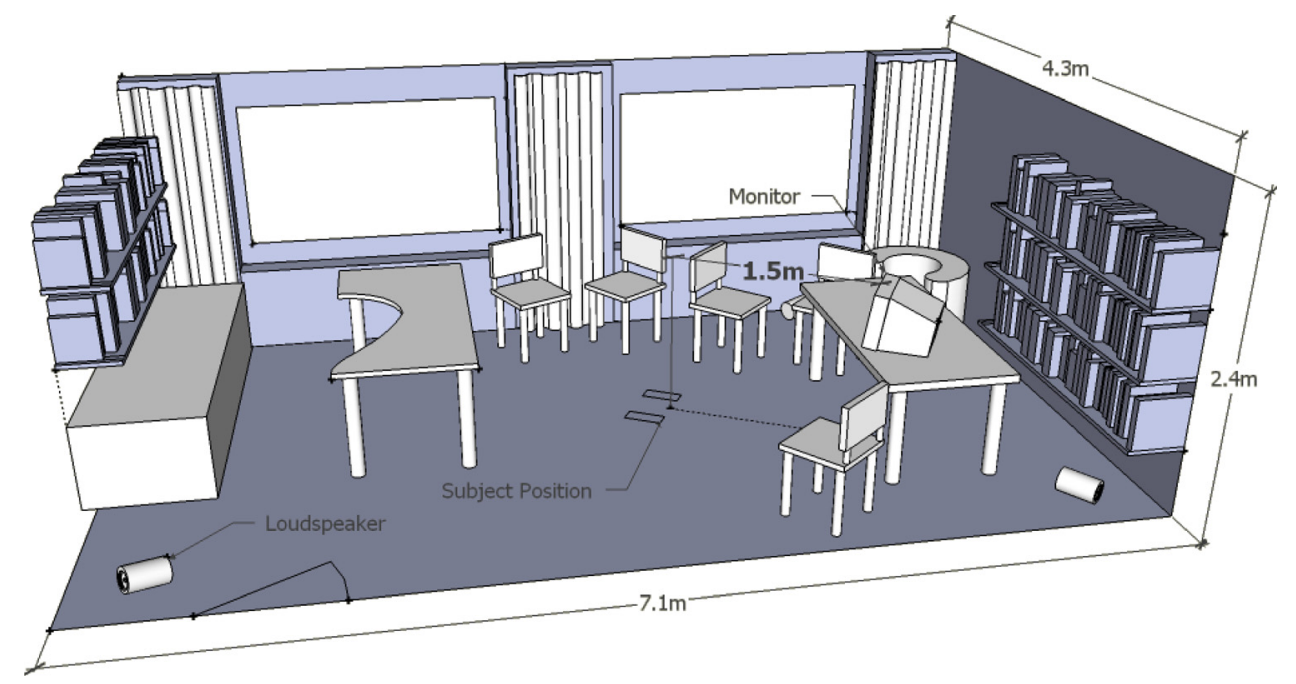

FIG. 3. (Color online) In situ room layout with dimensions and equipment locations.

type and level at the subject position. A separate file was created for each condition resulting in sixteen noise signals. Each calibrated signal served as the noise source for the respective condition during the experimental testing.

The signal for each condition was coordinated with an individual subject's script of spoken text and presented using a computer in an adjacent control room. The signal for each condition lasted for two minutes - the first minute was to acclimate the subject and measure the background noise, and the second minute was to measure the background noise in the presence of the subject's speech. This approach provided a precise, relative difference in measured level of the background noise with and without speech while accounting for the presence of the wearer's body, clothing, etc., in both measurements. The experimental setup required no active interaction by the test subject-they only had to speak the text as it was presented on the screen. This approach allowed the subject to respond freely to the testing environment. The script was displayed in the corresponding testing room using a 16 in. color monitor.

\section{Dosimeter}

The dosimeter used in this study was a Larson Davis Spark 705+ dosimeter, which meets the requirements of a Type 2 sound level meter as specified in ANSI S1.252002. ${ }^{27}$ (An ANSI Type 2 meter requires a tolerance of \pm 2 dB.) This model utilizes a prepolarized, electret microphone, and windscreen. The dosimeters were calibrated before and after the study with no necessary corrections. The meter settings were defined with a response time of "fast" (125 ms), averaging interval of $1 \mathrm{~s}$, exchange rate of 3 , and an $A$ weighting applied to the rms-pressure.

The dosimeter was located on the top of the subject's right shoulder at the end of the clavicle bone and top of the deltoid muscle. The diaphragm of the microphone was oriented away from the head and parallel to the median plane.

\section{Script-spoken text}

The subjects used scripts during the measurements to minimize the effect of the variability of spoken words upon the measured sound pressure levels. The scripts were generated from Hagerman's list (refer to Table II) designed for the Swedish language. ${ }^{28}$ For the purpose of this study, ten unique sentences were generated for each condition of every subject. The random generation assured no subject saw the same script as any other subject and required consistent concentration throughout the testing session on the part of the individual subjects.

The nature of the words within the list allowed for similar sentence difficulty. LIX (acronym for "läsbarhetsindex," which means readability index) is one index designed for Swedish and Danish text. The average LIX value for Hagerman's list is 33, which relates to fictional writing on an interpretative scale-above children's books and below newspaper text.

TABLE II. Hagerman's word list designed for Swedish and includes English translation.

\begin{tabular}{lllll}
\hline \hline \multicolumn{2}{l}{ Hagerman's original list } & & & \\
\hline Swedish: & & & & \\
Karin & gav & två & gamla & knappar \\
Britta & höll & tre & hela & bollar \\
Märta & ser & fyra & stora & vantar \\
Peter & köpte & sex & nya & pennor \\
Svante & lånar & sju & vackra & korgar \\
Jonas & ägde & åtta & mörka & skålar \\
Elsa & flyttar & nio & ljusa & mössor \\
Anna & visar & elva & fina & dukar \\
Bosse & har & tolv & lätta & ringar \\
Gustav & tog & arton & svarta & lådor \\
English Translation: & & & \\
Karin & gave & two & old & buttons \\
Britta & held & three & whole & balls \\
Märta & sees & four & big & gloves \\
Peter & bought & six & new & pencils \\
Svante & borrows & seven & nice & baskets \\
Jonas & owned & eight & dark & bowls \\
Elsa & moves & nine & light & caps \\
Anna & shows & eleven & fine & cloths \\
Bosse & has & twelve & light & rings \\
Gustav & took & eighteen & black & boxes \\
\hline \hline & & & & \\
\hline \hline
\end{tabular}




\section{Subjects}

Twenty-three, Swedish-speaking subjects (11 male and 12 female) served as participants whose data was analyzed for this study. Their ages ranged from 20 to 43 with a mean age of 26. Participants in the study answered a brief questionnaire prior to performing the test. The questions related to issues associated with vocal production and health and were as follows.

(1) Are you currently suffering from any respiratory illness?

(2) Have you recently noticed any difficulties speaking/ vocalizing?

(3) Do you smoke?

(4) Have you had any voice training?

Subjects with any positive (yes) responses were asked to elaborate. The results of these responses included one person experiencing residual effects at the end of a cold/flu and one person with allergy symptoms. No subjects declared any difficulties in speaking. Eight subjects had previous voice training, which included being a member of a choir, singing lessons, and sessions with a speech therapist during childhood. The affirmative responses to these questions were not associated with any outlying data.

Audiograms were conducted on each participant using the Oscilla USB-300 audiometer in conjunction with AUDIOCONSOLE software. The audiometric results of all twentythree subjects were hearing thresholds $\leq 25 \mathrm{dBHL}$ (decibel Hearing Level). A lower hearing level score relates to better hearing ability.

All subjects in the study were compensated for their time upon completion of the tasks and were informed of this prior to their signing up. The test took approximately one hour.

Although the subjects knew that the study involved acoustical components, they were not aware of the goal of the study or the hypothesized outcome. Subjects were told to read aloud the scripted sentences as if communicating with someone standing at the screen from which they were reading. That imaginary "receiver" was in the same type of environment (i.e., they were speaking with someone in the same room).

\section{Data analysis}

\section{Software}

The dosimeter data was obtained using the Larson Davis Spark $705+\mathrm{m}$ and was downloaded using the BLAZE software by Larson Davis. Under each condition, two independent $L_{p A \text {,eq }}$ values were calculated to determine the average sound pressure level over the course of each minute when the subject was not speaking $\left(L_{p A \text {,no speech }}\right)$ and when the subject was speaking $\left(L_{p A \text {,speech }}\right)$. The resulting values for each condition were used to calculate the contribution of speech, $C_{S}$, to the dosimeter measurement (i.e., the difference in measured sound pressure level as measured by the dosimeter when the subject was speaking and not speaking).

The speech contribution values associated with each condition were imported into SPSS v15.0.1 for statistical analysis and arranged by subject. Basic statistical analysis was performed along with applicable analysis of variance
(ANOVA) for further understanding of the main and interaction effects of variables.

\section{Preliminary analysis}

Preliminary statistical analysis was performed on the calculated contribution of speech, $C_{S}$. The purpose of the preliminary analysis was to perform an initial assessment of raw data and to verify the following assumptions necessary for parametric tests ${ }^{29}$-normal distribution, homogeneity of variance, interval data, independence, and sphericity.

\section{ANOVA}

A general linear model was created using ANOVA to investigate relationships between the experimental variables within the study as they relate to the speech contribution of subjects. Specifically, a factorial repeated-measures ANOVA general linear model was used to determine the statistical significance and interaction effects of the experimental variables. This method of analysis accounts for the following two major factors in the experimental design of the project: (1) within-subject design and (2) multiple independent variables (i.e., room, noise, and level).

In order to compare the group means, a test statistic, an $F$-ratio, was calculated to quantify the quality of fit of the model to the data. Significant results from this study are expressed with at least 0.05 significance $(p<0.05) .{ }^{29}$ Furthermore, effect size, $\omega^{2}$ (or $r$ depending on the analysis), was used to objectively measure the magnitude of the effect of each variable. Post hoc tests were used to make comparisons or contrasts between two groups to find specified statistical significance.

\section{RESULTS}

Statistical analysis of the data provided a quantifiable understanding of the effects of the speaker's voice on noise dosimeter measurements and also provided a means of accounting for speech effects in future dosimeter measurements. It was possible to use the results of the study to verify the measurement method with previous studies and investigate individual variables in the study.

\section{A. Lombard effect-Test method verification}

As a means of verifying the speaking conditions of the study, an analysis of the Lombard function (i.e., rate of change in speech level with a change in background level) was compared to previously published results. The mean speech level was calculated by logarithmically subtracting the energy of the measured level without speech from the measured level with speech. The calculated speech levels were then plotted by noise type across noise level and subdivided by room type. The four resulting curves are compared to average background levels and are shown in Fig. 4.

\section{B. Preliminary analysis}

In order to perform parametric tests it was necessary to verify normality of distribution, homogeneity of variance, 


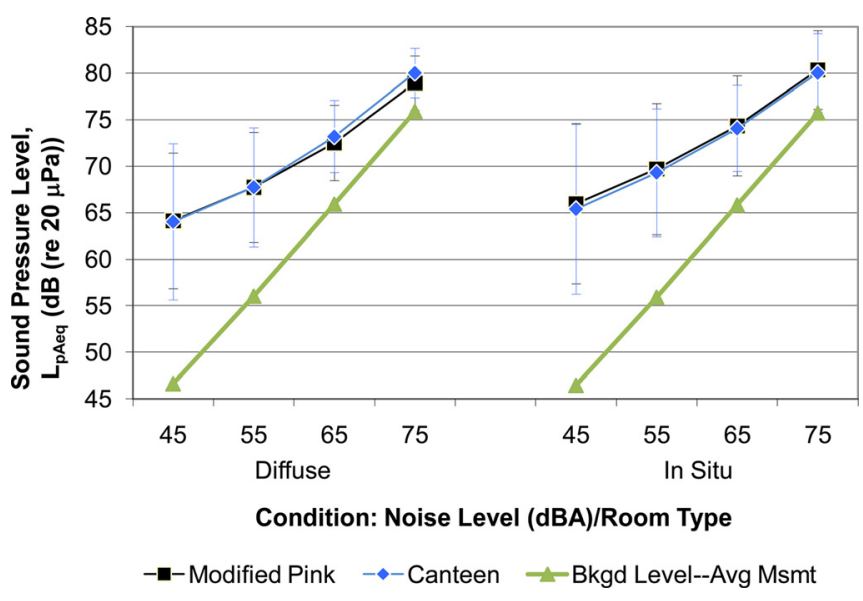

FIG. 4. (Color online) Calculated mean speech and background level by condition $(N=23)$. Background levels as measured by the dosimeter and averaged across subjects. Error bars denote $95 \%$ confidence interval for the mean speech level.

and sphericity of the data set from subjects with hearing thresholds $\leq 25 \mathrm{dBHL}$. The data set was found to be normally distributed for all sixteen conditions. Homogeneity of variance was violated across levels for three of the four room/noise categories. However, the violation was acceptable because ANOVA has proven to be a suitable statistical approach when sample sizes remain the same, which was the case for this analysis. ${ }^{29}$ For post hoc analysis, a GamesHowell correction was applied to account for the heterogeneity of variance. Three of the four applicable effects violated sphericity and required Greenhouse-Geisser correction factors-level; room and level; and room, noise, and level.

\section{Analysis of variance}

A test of within-subject effects indicates significance for all three main effects (Table III) and no significance for all interaction effects.

\section{Room type effect}

Further statistical analysis of the effects of the experimental variables began with the influence of room type upon the speech contribution to dosimeter measurements.

Table IV shows the mean and difference in mean between the two rooms as calculated across both noise types and all noise levels. A within-subject test concluded that there is a significant difference in speech contribution between the diffuse and in situ rooms, $F(1,22)=11.32$,

TABLE III. Test of within-subject main effects for the mean speech contribution level.

\begin{tabular}{lccc}
\hline \hline Independent variable & $F$-ratio & $p$-value & Effect size \\
\hline Room & $F(1,22)=11.32$ & 0.003 & $r=0.58$ \\
Noise & $F(1,22)=14.20$ & 0.001 & $r=0.63$ \\
Level $^{\mathrm{b}}$ & $F(1.2,25.9)=328.17$ & 0.000 & $r=0.97$ \\
\hline \hline
\end{tabular}

${ }^{\mathrm{a}}$ significant effect, $p<0.01$.

${ }^{\mathrm{b}}$ Greenhouse-Geisser correction applied to degrees of freedom for violation of sphericity.
TABLE IV. Room type effect-Mean, standard error, and difference in mean of speech contribution $(\mathrm{dB})$ for each room type. Mean values calculated as an average across noise type and noise level.

\begin{tabular}{lccc}
\hline \hline Room & $\begin{array}{c}\text { Mean } \\
(\mathrm{dB})\end{array}$ & $\begin{array}{c}\text { Std. error } \\
(\mathrm{dB})\end{array}$ & $\begin{array}{c}\text { Mean diff. } \\
(\mathrm{dB})\end{array}$ \\
\hline Diffuse & 10.59 & 0.48 & $1.34^{\mathrm{a}}$ \\
In situ & 11.93 & 0.57 & \\
\hline \hline
\end{tabular}

${ }^{a}$ Significant difference in mean, $p<0.01$.

$p<0.01, \omega^{2}=0.58$. Based on the effect size $\left(\omega^{2}=0.58\right)$, the effect of the room upon the outcome is large and is a significant source of variance of the data set. This finding was upheld by a pairwise comparison of the two rooms, and it was determined that the mean speech contribution is higher for the in situ room. The resulting mean difference is $1.34 \mathrm{~dB}$ and is significant, $p<0.01$.

A comparison of the mean levels of speech contribution for the two room types is shown in Fig. 5. The results are separated by noise type and displayed across noise level. Figure 5 shows the significance of the effect of room type on the mean speech contribution across conditions. The in situ room condition resulted in higher speech contribution across all noise levels regardless of the type of noise. At low levels, the difference in mean speech contribution is as much as $2.1 \mathrm{~dB}$ in modified pink noise. However, at higher levels, the influence of the room diminishes to less than $0.5 \mathrm{~dB}$ for the canteen noise.

\section{Noise type effect}

It was necessary to investigate the statistical significance of the effect of the noise type-modified pink and canteen. Table $\mathrm{V}$ shows the mean and difference in mean between the two noise types averaged across both room types and all noise levels.

Both the pairwise comparison and the within-subject test conclude that there is a significant difference in speech contribution between the modified pink noise and canteen noise used in the study, $F(1,22)=14.20, p<0.05, \omega^{2}=0.63$. Based on the effect size $\left(\omega^{2}=0.63\right)$, the effect of noise type is large and accounts for a significant portion of the variance in the data. The mean speech contribution calculated across

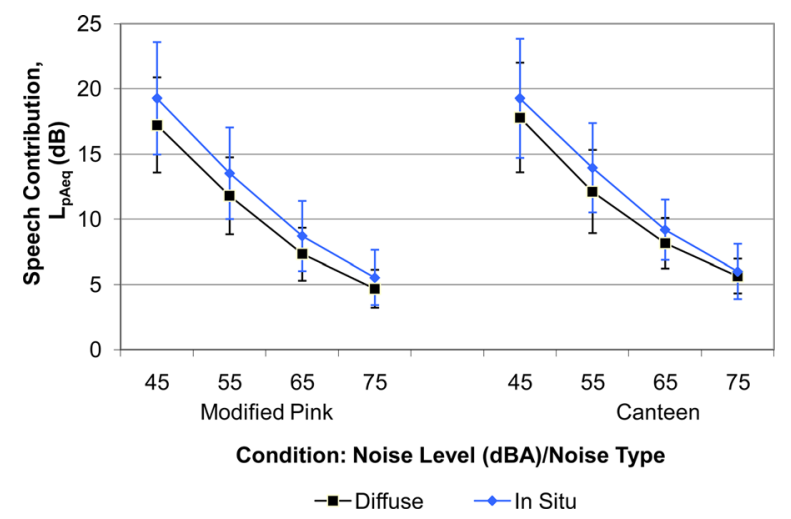

FIG. 5. (Color online) Comparison of mean speech contribution between room types-speech contribution to dosimeter measurements with error bars depicting \pm 1 standard deviation for each condition. 
TABLE V. Noise type effect-Mean, standard error, and difference in mean of speech contribution $(\mathrm{dB})$ for each noise type. Mean values calculated as an average across room type and noise level.

\begin{tabular}{lccc}
\hline \hline Noise type & $\begin{array}{c}\text { Mean } \\
(\mathrm{dB})\end{array}$ & $\begin{array}{c}\text { Std. error } \\
(\mathrm{dB})\end{array}$ & $\begin{array}{c}\text { Mean diff. } \\
(\mathrm{dB})\end{array}$ \\
\hline Modified pink & 11.01 & 0.490 & $0.51^{\mathrm{a}}$ \\
Canteen & 11.52 & 0.498 & \\
\hline \hline
\end{tabular}

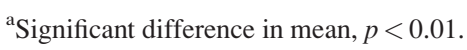

both room type and noise level is $0.5 \mathrm{~dB}$ higher for canteen noise than for the modified pink noise conditions.

The significance of the noise type effect upon mean speech contribution can be more closely investigated across condition by examining Fig. 6 . The results are organized by room and noise level showing a direct comparison of the mean speech contribution for both noise types. For this comparison, it is important to note the similarities in the results from the two noise types within each room. The differences in speech contribution between noise types at a given noise level is less than $1.0 \mathrm{~dB}$ in the diffuse room and less than $0.5 \mathrm{~dB}$ for the in situ room. Even though these differences between noise type are small $(<1.0 \mathrm{~dB})$, statistically the results are considered highly significant with a large effect upon mean speech contribution.

\section{Noise level effect}

The final individual effect to investigate was noise level. The previous analyses in this study have shown an obvious inverse relationship between noise level and speech contribution. However, it was important to verify the statistical significance of the effect using parametric analysis and to pursue further investigation with post hoc tests.

Table VI shows the mean and difference in mean between consecutive noise level conditions across both room types and noise types.

A significant difference was found between noise levels through a within-subject test using a Greenhouse-Geisser correction due to lack of sphericity, $F(1.2,25.9)=328.17$, $p<0.05, r=0.97$. This effect size is the largest of the three independent variables suggesting that noise level has the

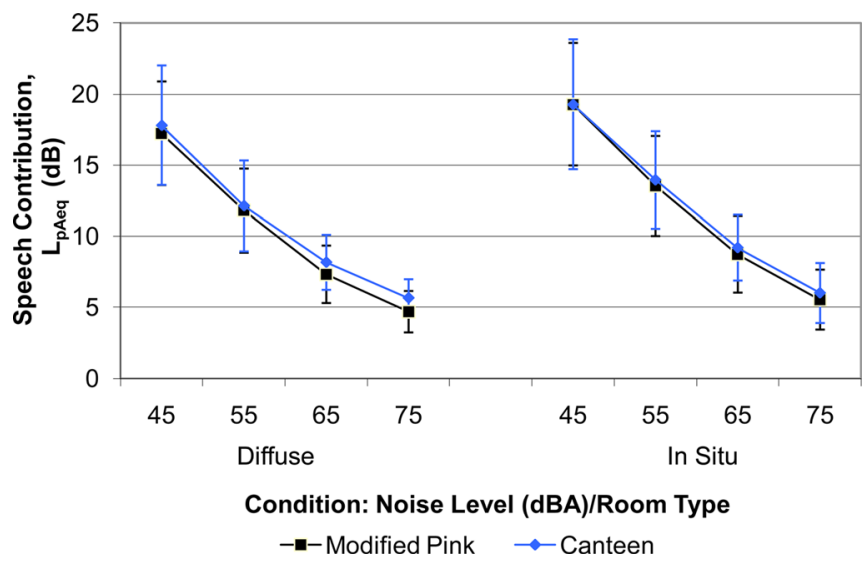

FIG. 6. (Color online) Comparison of mean speech contribution between noise types - speech contribution to dosimeter measurements with error bars depicting \pm 1 standard deviation for each condition.
TABLE VI. Noise level effect-Mean and standard error of speech contribution $(\mathrm{dB})$ for each noise level along with difference in mean $(\mathrm{dB})$ between consecutive noise levels. Mean values calculated from average across room type and noise type.

\begin{tabular}{lccc}
\hline \hline Noise level & $\begin{array}{c}\text { Mean } \\
(\mathrm{dB})\end{array}$ & $\begin{array}{c}\text { Std. error } \\
(\mathrm{dB})\end{array}$ & $\begin{array}{c}\text { Mean diff. } \\
(\mathrm{dB})\end{array}$ \\
\hline 45 & 18.4 & 0.77 & $5.5^{\mathrm{a}}$ \\
55 & 12.9 & 0.60 & $4.6^{\mathrm{a}}$ \\
65 & 8.3 & 0.42 & $2.8^{\mathrm{a}}$ \\
75 & 5.5 & 0.34 & \\
\hline \hline
\end{tabular}

${ }^{\text {a }}$ Significant difference in mean, $p<0.01$.

greatest influence upon the outcome of the speech contribution to the dosimeter measurement.

Further investigation of these findings was conducted using a pairwise comparison of each level to all the other levels. The calculated mean differences between noise levels were all statistically significant and are shown in Table VII. These results confirm the within-subject test and specify that each level's mean speech contribution is significantly different from all other levels from 45 to $75 \mathrm{dBA} L_{\mathrm{eq}}$.

Since the noise level variable included four levels, unlike the two possible categories within room type and noise type, it was possible to conduct a trend analysis. This analysis investigated the statistical likelihood that the data fit a particular polynomial trend (i.e., linear, quadratic, etc.) The result of the polynomial contrast was a significant linear trend, $F(1,22)=355.4, p<0.05, r=0.97$ and a significant quadratic trend, $F(1,22)=88.4, p<0.05, r=0.89$. Figure 7 clearly shows the inverse relationship between speech contribution and noise level with an approximate $0.5 \mathrm{~dB}$ decrease in speech contribution for every $1 \mathrm{~dB}$ increase in noise level. In addition, one can see that the standard deviation has an inverse relationship to noise level.

Further investigation of the trends looked at the difference in speech contribution between noise levels. Figure 8 shows the difference in mean speech contribution between levels (from the top row of data in Table VII) and compares it to a constant change in speech contribution. If the data were linear, the change in speech contribution from one noise level to the next would remain constant (constant

TABLE VII. Difference in means of speech contribution (dB) between each noise level. Mean values calculated from average across room type and noise type.

\begin{tabular}{|c|c|c|c|c|c|}
\hline & & \multicolumn{4}{|c|}{ Mean difference $^{\mathrm{a}}(a-b)$} \\
\hline & & \multicolumn{4}{|c|}{$b$} \\
\hline & & 45 & 55 & 65 & 75 \\
\hline & 45 & & 5.5 & 10.0 & 12.9 \\
\hline \multirow[t]{3}{*}{$a$} & 55 & -5.5 & & 4.5 & 7.4 \\
\hline & 65 & -10.0 & -4.5 & & 2.8 \\
\hline & 75 & -12.9 & -7.4 & -2.8 & \\
\hline
\end{tabular}

${ }^{\mathrm{a}}$ All results significant, $p<0.01$. 


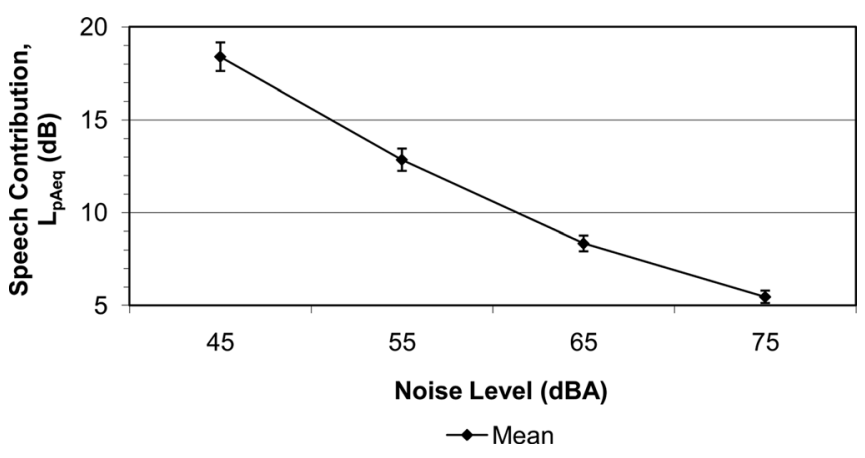

FIG. 7. Mean speech contribution by noise level—averaged across noise type and room type. Error bars depict \pm 1 standard deviation.

slope) as shown with the "linear extrapolation." The actual change in mean speech contribution deviates from linear, which suggests a diminishing influence of the Lombard effect and illustrates the quadratic trend.

\section{DISCUSSION}

\section{A. Lombard effect}

The physical response of increasing one's voice with respect to changes in background sound level and auditory feedback, the Lombard effect, has been studied multiple times over the past 100 years. Those studies have produced sets of Lombard functions used to quantify the Lombard effect. These Lombard functions as summarized by Lane and Tranel ${ }^{19}$ for studies prior to 1971 and also a recent investigation by Giguère et $a l .{ }^{30}$ contain linear approximations of $0.2-0.6 \mathrm{~dB} / \mathrm{dB}$ for background sound pressure levels of $45-75 \mathrm{~dB}$.

The calculated linear approximations from the current study agree with previous results for the mid-range noise levels with between 0.49 and $0.54 \mathrm{~dB} / \mathrm{dB}$ as defined by the room type and noise type. Applied generally, this study indicates that an individual will increase the level of their voice approximately $0.5 \mathrm{~dB}$ for every increase of $1.0 \mathrm{~dB}$ in background noise level within the range of 45-75 dBA $L_{\mathrm{eq}}$.

By verifying the Lombard effect results in this study with previous work, it was possible to expand the application of the Lombard effect to the specific effects of speech on dosimeter measurements.

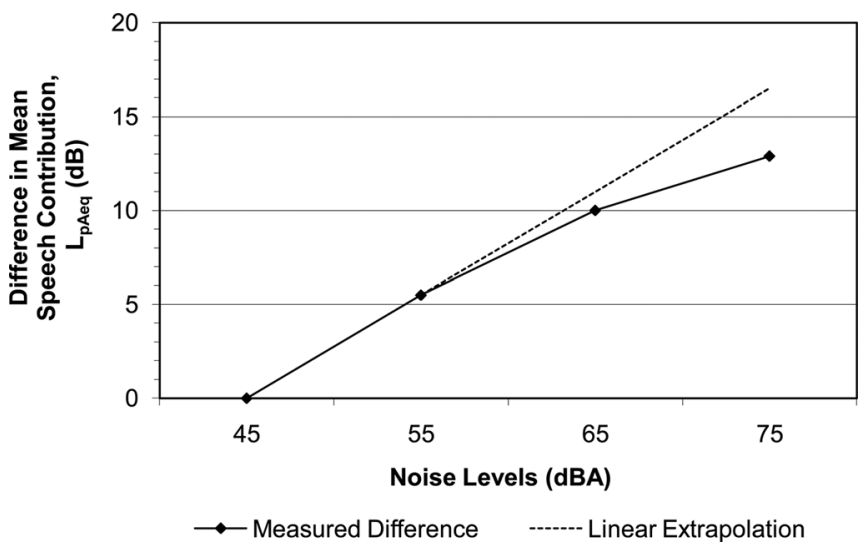

FIG. 8. Change in mean speech contribution across noise level. The rate of change in mean speech level decreases as noise level increases.

\section{B. Room type effect}

A diffuse room is often thought of as an inherently louder acoustic environment due to energy remaining in the space longer. Since the background noise levels were calibrated to the same level in both rooms, one might expect the measured speech contribution to be higher in the diffuse room rather than the in situ room because energy from the voice would remain in the reverberant space longer due to less absorption.

Higher speech contribution in the diffuse room assumes that the subject will produce the same level of speech in both environments. Contrary to this view, the results show that the in situ environment produces higher speech contribution levels and can be explained by the Lombard effect.

The Lombard effect presents two possible influences on a person's vocal effort - the ambient noise level and the auditory feedback of the speaker's voice. Calibration of the signals in both rooms excluded the influence of the ambient noise level as the cause of any difference in speech contribution between rooms. Therefore, the higher mean levels of speech contribution in the in situ room is likely related to less auditory feedback, or fewer perceived reflections, of the subject's voice in the in situ space. Fewer reflections are to be expected in a space with more absorption. In accordance with the Lombard effect, if the subject perceives fewer reflections in a room with absorption, the subject will naturally respond by increasing their vocal effort and, in turn, increasing the speech contribution to the measured levels.

\section{Noise type effect}

An explanation for the overall difference between modified pink noise and canteen noise could be related to the temporal characteristics of each noise. The instantaneous sound pressure levels within the canteen noise are highly timevariant. Despite multiple groups of conversations and the application of sound level compression to the signal, instantaneous sound pressure levels would increase and decrease around the calibrated, equivalent noise level for the condition. In contrast to the canteen noise, the modified pink noise signal was relatively constant in level. The time-variant characteristic of the level for the canteen noise could be responsible for the increase in speech contribution as a subject may tend to "over-compensate" for a brief rise in background sound level.

Furthermore, the higher speech contribution in the canteen noise could be a result of the subject's goal to ensure communication. Subjects may experience increased competition for understanding when competing with other voices in the canteen noise. The heightened sense of competition could result in increased vocal effort and increased speech contribution compared to the modified pink noise.

\section{Noise level effect}

It is of interest to confirm the dominance of the effect of noise level on the contribution of speech due to the Lombard effect; however, a trend analysis provides better understanding of the intricacy of the Lombard effect. The confirmation 
of a quadratic trend suggests the body's inability to consistently compensate for increased noise levels as it approaches the maximum vocal effort. The remaining strong linear trend is likely a result of the variability across subjects combined with the limited range of noise levels. A study of more subjects across a greater range of noise levels would likely strengthen the quadratic trend and weaken the linear trend.

Additionally, it is interesting to note the inverse relationship between standard deviation and noise level. Throughout Figs. 5-7, the standard deviation can be seen decreasing as noise level increases. This result suggests that as the subjects reach their limit of vocal effort, the individual variability among subjects is reduced.

\section{E. Interaction effects}

The non-significant results of the interaction effects suggest that there is no complex relationship between variables. The influences on the subjects can be solely explained by the individual experimental variables. To find significant interactive effects, one looks for non-parallel lines in the plots of the corresponding individual variables. In the case of this study, all variables plotted as nearly parallel lines which demonstrate the lack of interactive effects.

\section{F. Application of results}

In order to develop a method of accounting for a wearer's voice in body-mounted noise dosimeter measurements, it is possible to apply the statistical results from this study. Analysis of the independent variables shows all three experimental variables having significant effects upon the speech contribution to dosimeter measurements. However, if one looks at the mean difference within each variable independently, one will notice the mean difference due to room type $(1.3 \mathrm{~dB})$ and noise type $(0.5 \mathrm{~dB})$ is less than the tolerance of a type 2 sound level meter $( \pm 2 \mathrm{~dB})$ commonly used for dosimetry. The remaining experimental variable influencing the speech contribution of dosimeter measurements is the background noise level with a mean difference ranging from 2.8 to $5.5 \mathrm{~dB}$ which exceeds the tolerance of a type 2 sound level meter.

The final main factor that must be accounted for when applying the results beyond this study is the amount of time a subject speaks during the measurement period. This factor was controlled in this study, but in natural settings, speech time is highly variable-depending on occupation, task, and individual speaking tendencies. However, if one can estimate the percentage of speaking time during the day (e.g., through a survey or self-assessment of the dosimeter wearer), it is possible to also estimate the effect of the wearer's voice on the dosimeter measurement.

The data obtained from this study allows for the development of a set of curves to approximate the speech contribution of the measured dosimeter level based on a given room's background noise level and an estimated percentage of speaking time. Figure 9 shows speech contribution (in $\mathrm{dB})$ due to the wearer's voice in the presence of background noise and with respect to the percentage of speaking time. The contours in Fig. 9 were developed based on the average measured sound pressure level in the presence of the wear-

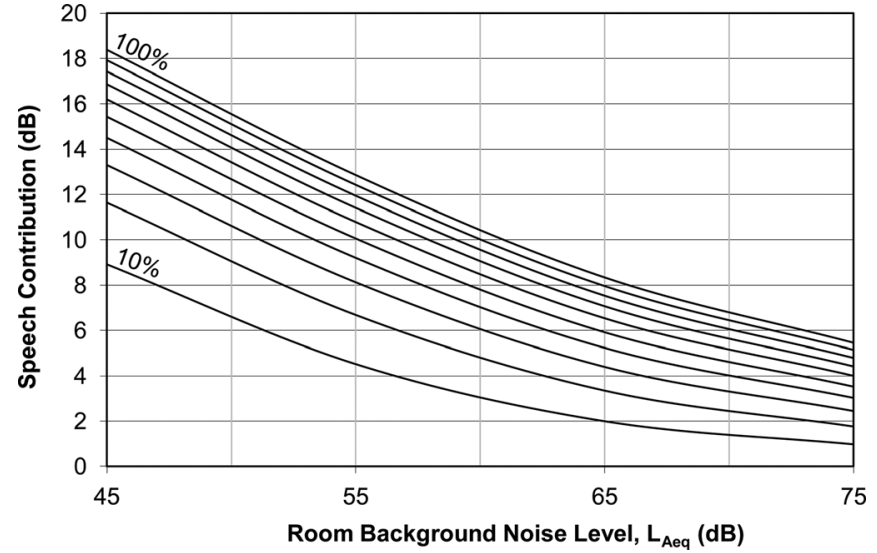

FIG. 9. Contribution of speech $(\mathrm{dB})$ to a dosimeter measurement due to a wearer's voice in the presence of background noise. Speech contribution can be determined based on the room background noise level (without speech) and percentage of speaking time. The contours define the percentage of speaking time from 10 to $100 \%$ in medium-level noise environments from 45 to $75 \mathrm{dBA}$.

er's speech for each background level-averaged across both room type and noise type. The $100 \%$ contour corresponds to the speech contribution values from Fig. 7. The remaining contours are calculated based on the timeweighted average level of the two sources (i.e., the measured background noise and the measured level with the wearer's voice at the dosimeter). To calculate speech contributions, $C_{S}$, in Fig. 9, Eq. (1) becomes

$$
C_{S}=L_{n}-L_{p A, \text { no speech }}
$$

where $L_{n}$ is the calculated $A$-weighted equivalent sound pressure level based on the percentage of speaking time, $n$,

$$
\begin{aligned}
L_{n}= & 10 \log \left[\left(10^{L_{p A, \text { no speech }} / 10}\right)\left(1-\frac{n}{100}\right)\right. \\
& \left.+\left(10^{L_{p A, \text { speech }} / 10}\right)\left(\frac{n}{100}\right)\right] .
\end{aligned}
$$

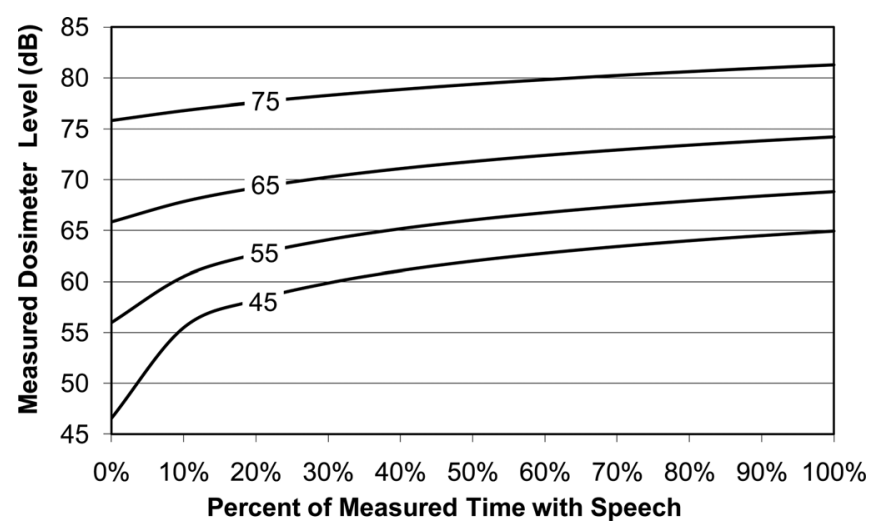

FIG. 10. The contours represent the estimated room background sound pressure level (dBA) based on a dosimeter level measurement and the percentage of measured time with speech. The difference between the estimated room background level and the dosimeter level is due to the contribution of speech on the dosimeter measurement by the wearer's voice and the effect of a body-mounted measurement. 
Similarly, a set of curves can also be developed to compare the room background sound pressure level to the measured dosimeter level based on the percentage of speaking time (Fig. 10). While Fig. 9 provides the results in terms of speech contribution, Fig. 10 provides a direct method of determining the room background sound level given a dosimeter measurement and the estimated time of speech. For Fig. 10, the calculated level based on the percentage of speaking time, $L_{n}$, is plotted across background noise level to generate the resulting curves.

The application of the proposed method can be used in situations when it is necessary to obtain sound pressure levels for an individual's noise environment while excluding the effect of the wearer's voice. Such situations could occur when monitoring someone's noise exposure in a mediumlevel acoustic environment or if one is interested in annoyance due to factors other than self-noise.

The method can potentially be useful in medium-level background noise environments such as hospitals, schools, office spaces, etc. As an example application: in hospitals, dosimeters have several advantages over stationary meters because the staff frequently moves from one room to another. However, as discussed previously, dosimeter level readings made in hospitals are not necessarily indicative of the room background noise because of the large contribution of the wearer's voice.

Figures 9 and 10 are indicative of how the results of this study could be applied. For example, Fig. 9 could be used to estimate the specific contribution of speech on dosimeter measurements or Fig. 10 can be used to estimate the background noise level based on measured dosimeter levels. However, we acknowledge the fact that the curves may need additional validation for other populations and contexts not examined in this study.

An example of the use of Fig. 9 can be found in previous research by Ryherd, Persson Waye, and Ljungkvist. ${ }^{31}$ The authors made measurements in a neurological intensive care unit (ICU) documenting a difference of $12-13 \mathrm{~dB}$ between mounted dosimeter measurements on nurses and stationary sound level meters $\left(L_{p A \text {,eq }}=53\right.$ to $\left.58 \mathrm{~dB}\right)$. This difference in measured level between meters is plausible based on the results from the current study. Using Fig. 9, a background level of $55 \mathrm{~dB} L_{p A \text {,eq }}$ and a speech time of $50 \%$ would give a contribution of $10 \mathrm{~dB}$ to the dosimeter level. The remaining difference of $2-3 \mathrm{~dB}$ can possibly be explained by the nurse's activities and their proximity to the dosimeters as compared to the location of the stationary measurement.

This method of determining the speech contribution would be complicated by situations where the background noise level varies considerably. Such a situation would require analysis of the dosimeter's time history to develop a histogram of time exposure to discrete level intervals. Specific analysis would also be necessary to determine if speech is equally likely to occur in all level intervals. Each level interval could then be analyzed individually for speech contribution and then summed using a time-weighted average of the calculated sound levels.

This method was developed using a quantity of subjects that ensured statistical power in order to calculate the Lom- bard slope under these controlled conditions. This method of accounting for speech contribution should be applied to a sample size that also has adequate statistical power. Conditions or subjects that result in a lower Lombard slope will cause the contours in Figs. 9 and 10 to compress in the higher background noise levels. In other words, the influence of a wearer's voice on dosimeter measurement would be less at higher background levels if the rate at which speakers increase their speech level is reduced.

The proposed method only accounts for one of the experimental variables investigated in this study-noise level. To account for the effect of room type and noise type, one can apply a potential variability of the measurement of up to $\pm 0.9 \mathrm{~dB}$ which is a total variability range of $1.8 \mathrm{~dB}$ - the summation of the two average speech contributions due to room type and noise type. If, for instance, measurements were conducted in a room with some absorptive surfaces and the background noise consisting of speech, the predicted levels could potentially have a variation of $0.9 \mathrm{~dB}$.

\section{CONCLUSION}

This work has demonstrated the effects of the wearer's voice as a dominant sound source on body-mounted noise dosimeters in medium-level noise environment. Subjects experienced a controlled set of acoustic conditions by varying room type, noise type, and noise level. Data obtained from the body-mounted dosimeter measurements with and without speech was analyzed to determine statistical significance of the experimental variables. Based on the analysis, it was possible to develop a method to account for a wearer's speech and to improve dosimetry measurements in mediumlevel noise environments.

Based upon the within-subject tests and the pairwise comparisons, it can be concluded that the experimental variables of room type, noise type, and noise level have a statistically significant effect upon the contribution of speech to a dosimeter measurement. However, the results of this study show no statistically significant interaction effects among the same experimental variables. Noise level was shown to have the greatest effect upon speech contribution-a change in speech contribution by as much as $5.5 \mathrm{~dB}$ between sequential levels. Conversely, noise type appeared to have the least effect upon speech contribution-only a change of $0.5 \mathrm{~dB}$ between types.

After reviewing the results, a method was proposed to account for a wearer's speech in body-mounted dosimeter measurements by focusing on background noise level and the percentage of speaking time during a given time period. Depending on the desired outcome, this method can be applied to determine the speech contribution of a dosimeter measurement or can be applied to estimate the room background noise level based on dosimeter measurements.

Further studies can be used to verify the theoretical model developed in this investigation including various occupational environments and measurement locations (e.g., near the ear). This could include expansion into cases where the background noise is above $75 \mathrm{dBA}$. Metrics aside from $L_{A \text { eq }}$, such as dose-type metrics could also be analyzed. 
Additionally, the acoustic content of the background noise signal was not addressed as a specific variable in this study aside from the general "modified pink" and "canteen" noise designations. Future research could more specifically analyze how spectral content, tonality, and time-variance, etc., of the background noise impacts results.

\section{ACKNOWLEDGMENTS}

This work was supported by the Fulbright Scholar Program, the Swedish Fulbright Commission, and the Acoustical Society of America's Hunt Fellowship. We are also grateful to Börje Wijk at the Department of Applied Acoustics at Chalmers University for his technological assistance.

${ }^{1}$ W. A. Yost, N. Koita, R. Maslo, P. Patel, R. Patel, and B. Ringhouse, "Dosimeter measures of sound exposure experienced by university students," J. Acoust. Soc. Am. 120(5), 3163 (2006).

${ }^{2}$ D. Cheenne, C. Lee, M. Cappiello, S. Lalk, C. Martin, and P. Muzzy, "A comprehensive noise study for the city of Lincoln, Nebraska," J. Acoust. Soc. Am. 120(5), 3336 (2006).

${ }^{3}$ R. Neitzel, N. Seixas, J. Olson, W. Daniell, and B. Goldman, "Nonoccupational noise: Exposures associated with routine activities," J. Acoust. Soc. Am. 115(1), 237-245 (2004).

${ }^{4}$ E. H. Berger and W. Kieper, "Representative 24-h Leqs arising from a combination of occupational and nonoccupational noise exposures," J. Acoust. Soc. Am. 95(5), 2890-2891 (1994).

${ }^{5}$ L. H. Royster and J. D. Royster, "Nonoccupational noise exposures and their estimated daily L (eqs.) for general commercial air travel and local bars, restaurants, discos, and socialization establishments," J. Acoust. Soc. Am. 95(5), 2891 (1994).

${ }^{6} \mathrm{G}$. F. Kuhn, "The pressure transformation from a diffuse sound field to the external ear and to the body and head surface," J. Acoust. Soc. Am. 65(4), 991-1000 (1979).

${ }^{7}$ J. Asztely and M. Kleiner, "On the placement of personal noise meter microphones," Proc. Inter. Noise 81, 851-856 (1981).

${ }^{8}$ D. A. Giardino and J. P. Seiler, "Interaction of body-worn measurement microphones with the undisturbed sound field," J. Acoust. Soc. Am. 100(4), 2672 (1996).

${ }^{9}$ G. F. Kuhn, "Comparisons between A-weighted sound-pressure levels in the field and those measured on people or manikins," J. Acoust. Soc. Am. 79(S1), S2 (1986).

${ }^{10}$ M. P. Valoski, J. P. Seiler, M. A. Crivaro, and G. Durkt, "Comparison of noise exposure measurements conducted with sound level meters and noise dosimeters under field conditions," U. S. Department of Labor, Informational Report No. 1230, Mine Safety and Health Administration, 1995.

${ }^{11}$ D. A. Giardino and J. P. Seiler, "Uncertainties associated with noise dosimeters in mining," J. Acoust. Soc. Am. 100(3), 1571-1576 (1996).
${ }^{12}$ L. H. Royster and J. D. Royster, "Types and frequencies of abnormal sound levels when using noise dosimeters to establish worker TWAs," J. Acoust. Soc. Am. 103(5), 2921(1998).

${ }^{13}$ S. Shackleton and M. D. Piney, "A comparison of two methods of measuring personal noise exposure,” Ann. Occup. Hyg. 28(4), 373-390 (1984).

${ }^{14} \mathrm{P}$. Sriwattanatamma and P. Breysse, "Comparison of NIOSH noise criteria and OSHA hearing conservation criteria," Am. J. Ind. Med. 37, 334-338 (2000).

${ }^{15}$ ANSI S12.19-1996, "Measurement of occupational noise exposure" (American National Standards Institute, New York, 2006).

${ }^{16}$ F. Lindstrom, A. C. Ohlsson, K. Persson Waye, and I. Claesson, “A pilot study of acoustic measurements of pre-school children and female preschool teachers' voices," Inter-Noise 2007, Istanbul, Turkey, August, 2007.

${ }^{17}$ S. Ternström, M. Bohman, and M. Södersten, "Loud speech over noise: Some spectral attributes with gender differences," J. Acoust. Soc. Am. 119(3), 1648-1665 (2006).

${ }^{18}$ A. Wakao, K. Takeda, and F. Itakura, "Variability of Lombard effects under different noise conditions," Proceedings of the International Conference on Spoken Language Processing (ICSLP) '96, Philadelphia, PA, 1996, Vol. 4, pp. 2009-2012.

${ }^{19}$ H. Lane and B. Tranel, "The Lombard sign and the role of hearing in speech,” J. Speech Hear. Res. 14, 677-709 (1971).

${ }^{20}$ W. Van Summers, D. B. Pisoni, R. H. Bernacki, R. I. Pedlow, and M. A. Stokes, "Effects of noise on speech production: Acoustic and perceptual analysis," J. Acoust. Soc. Am. 84(3), 917-928 (1988).

${ }^{21}$ J. B. Tufts, and T. Frank, "Speech production in noise with and without hearing protection,” J. Acoust. Soc. Am. 114(2), 1069-1080 (2003).

${ }^{22}$ F. Bozzoli and A. Farina, "Measurement of Active Speech Level inside cars using throat-activated microphone," Convention Paper, Audio Engineering Society, Berlin, Germany, 2004.

${ }^{23}$ S. Granqvist, "The self-to-other ratio applied as a phonation detector for voice accumulation," Logoped. Phoniatr. Vocol. 28(2), 71-80 (2003).

${ }^{24}$ J. Svec, P. S. Popolo, and I. R. Titze, "Measurement of vocal doses in speech: Experimental procedure and signal processing," Logoped. Phoniatr. Vocol. 28, 181-192 (2003b).

${ }^{25}$ P. E. Giua, C. D. Messino, and D. L. Johnson, "Elimination of the influence of wearer's voice by two-dosimeter system," J. Acoust. Soc. Am. 92(4), 2382 (1992).

${ }^{26}$ ANSI S1.4-1983, "Specifications for Sound Level Meters" (American National Standards Institute, New York, 2006).

${ }^{27}$ ANSI S1.25-1991, "Specifications for Personal Noise Dosimeters" (American National Standards Institute, New York, 2007).

${ }^{28}$ B. Hagerman, "Sentences for testing speech intelligibility in noise," Scand. Audiol. 11, 79-87 (1982).

${ }^{29}$ A. Field, Discovering Statistics Using SPSS, 2nd ed. (Sage Publications, London, England, 2005).

${ }^{30}$ C. Giguère, C. Laroche, É. Brault, J. Ste-Marie, M. Brosseau-Villeneuve, B. Philippon, and V. Vaillancourt, "Quantifying the Lombard effect in different background noises," J. Acoust. Soc. Am. 120(5), 3378 (2006).

${ }^{31}$ E. Ryherd, K. Persson Waye, and L. Ljungkvist, "Characterizing noise and perceived work environment in a neurological intensive care unit," J. Acoust. Soc. Am. 123(2), 1069-1080 (2008). 